\title{
Clinical Blood Draws: When Do They Require IACUC Approval?
}

Dr. David Burbank, an assistant professor at Great Eastern University, was excited about his planned study. However, at the 11th hour, he realized that a small amount of blood taken from four or five dogs would provide important additional preliminary data to support his pending NIH grant submission. Unfortunately, the school's animal facilities were not housing dogs at the time. So, acting on a suggestion from one his technicians, Burbank called the veterinarian at the local humane society and asked him if it was possible to obtain a small amount of freshly drawn blood from a few healthy-looking dogs. The veterinarian said that they routinely took blood samples for clinical purposes, and he saw no real problem in taking one additional milliliter from some of the larger animals to help Burbank's research. This was great news to Burbank, and with the veterinarian's assistance the blood was drawn, the data were gathered and analyzed, and the grant was submitted.

Under the new NIH 'just-in-time' procedures for peer review, Burbank's grant application was reviewed, received a favorable priority score, and was likely to be within the fundable range. He submitted to the Great Eastern IACUC an application that included the use of cats (the primary species of interest) and dogs (based on his supplementary data). The application used the words "based on preliminary data from dogs," a point missed during the protocol review process because dogs were occasionally housed at Great Eastern. After approval from the IACUC, Burbank began his research, but not everything went as planned. There were a series of small technical problems, and as a result some of the dogs did not appear as healthy as others, although they all eventually did well. Therefore, it was not surprising when Dr.
Shana Madela, the USDA Veterinary Officer, asked to see Burbank's protocol after observing some of the dogs during a regular inspection. Madela reviewed the promptly produced protocol, which clearly stated that no health or welfare problems were anticipated. Madela then asked to see the protocol that supported the "preliminary data from dogs," which, of course, did not exist. Burbank received a call, and he explained exactly what had happened. Madela said she would have to cite Great Eastern for having performed research without an IACUC-approved protocol. In its own defense, Great Eastern's IACUC Chairperson argued that it was just a little extra blood taken from clinical cases that had never needed an IACUC protocol to begin with. Additionally, it came from animals at a private humane organization that did no research at all. Madela was unimpressed. Her opinion was that blood was taken from a USDA-covered species for research purposes, whether it was a just a supplement to a clinical procedure or not.

What do you think? Did Burbank need the approval of the Great Eastern IACUC for the private veterinarian to take a little extra blood, or is Madela wrong in her interpretation?

\section{IACUC in a Bind Thanks to Lack of Information}

Stacy Pritt, DVM, and J. Fred Nostrant, BS

The above scenario accurately demonstrates that Great Eastern's IACUC should have requested additional information on the "based on preliminary data from dogs" statement that was included in the investi- gator's IACUC protocol. At that point, Great Eastern's IACUC could have learned of the research activities that generated the referenced data and started internal discussions, possibly with regulatory insight from the USDA, on how to handle such activities. However, because this interaction did not occur, the IACUC finds itself in a bind with the USDA.

A review of the Animal Welfare Regulations makes it clear that the IACUC's responsibility includes having "...review of activities involving animals," with animals being defined as "any live or dead dog, cat, nonhuman primate, guinea pig, hamster, rabbit, or any other warmblooded animal, which is being used, or is intended for use for research, teaching, testing, experimentation, or exhibition purposes"1. Because the investigator's research does include activities involving animals, there should have been some provision for IACUC review.

It is assumed that because the humane society is an off-site, private, and unaffiliated entity, Great Eastern's IACUC would have no authority over it. Some form of IACUC oversight and registration as a research facility would have therefore been necessary at the humane society. Because the humane society does not have an IACUC, there has been no IACUC review, approval, or oversight for this particular use of animals. Another consideration is that without an animal use protocol covering the bleeding procedure, there is no guarantee that the dogs would be bled only for clinical purposes rather than for a specific research purpose.

Had these activities been identified previously at the University's IACUC, someone could have suggested the alternative of ordering dog blood products from a commercial supplier. The disease-free status of

"Protocol Review" continues on p.20 
"Protocol Review" continues from p.17

such products could have eliminated potentially significant research variables, and the regulatory issues could have been avoided.

To help avoid situations similar to this one in the future, advanced protocol review training for IACUC members and training on animal welfare rules and regulations, along with protocol writing, for investigators would be in order.

\section{Reference \\ 1. USDA APHIS. 9 CFR, Subchapter A - Animal Welfare, Parts 1-3.}

Pritt is Director, Animal Resources Facility, Joslin Diabetes Center, Boston, MA. Nostrant is an IACUC Member, Elm Hill Breeding Labs, Chelmsford, MA and a Second Year Veterinary Student, Tufts University School of Veterinary Medicine, North Grafton, MA.

\section{Grant Deadline No Excuse}

Paula Samalonis, BA, CAHT, RLATG, and Beth Lotocki, MLAS, MT(ASCP)

It seems that Burbank overlooked proper procedures as he got caught up in the rush of the deadline for grant application submission. After all, the dogs were not 'research animals', and taking an extra milliliter of blood surely would not affect the well-being of the animals. Even though the dogs reside at the local humane society, they are covered under the AWA. Great Eastern receives funding from $\mathrm{NIH}$ and therefore must follow the Public Health Service (PHS) Policy. The Animal Welfare Regulations state the following as one of the responsibilities of the IACUC: "IACUC review of activities involving animals." Additionally, the PHS Policy states that " $[\mathrm{t}]$ his policy is applicable to all PHS-conducted or supported activities involving animals, whether the activities are performed at a PHS agency, an awardee institution or any other institution and conducted in the United States..." A recent comment from the Office of Protection from Research Risks (OPRR)/NIH (now the Office of Laboratory Animal Welfare, OLAW) regarding activities using animals, whether only to obtain blood or blood products, states that "PHS Policy applicability is not limited to research. It also includes all activities involving animals including testing and teaching ${ }^{3}$." Burbank obtained this blood specifically to analyze and collect data related to his proposed research project. OPRR/NIH states that, although these activities are not part of specific research protocols, "their use for these purposes contributes significantly to the institutional research program and constitutes activities involving animals ${ }^{3}$." Finally, the statement says, " $[\mathrm{t}]$ he IACUC must receive and approve of protocols and appropriate systems to monitor the use of animals prior to the commencement of such activities ${ }^{3}$." The IACUC Handbook also uses this point to answer a similar question about the necessity of IACUC approval for sentinel animals or blood donors ${ }^{4}$. Burbank is not in an unfamiliar situation. Rules on blood donor animals often lie in gray areas. The IACUC Handbook recommends that each institution develop a written policy on the procedures to follow when using donated blood $^{5}$. Whereas a full protocol may not be necessary, this leads to some form of paperwork that the IACUC needs to generate and review for approval to remain consistent with federal guidelines ${ }^{5}$.

In our opinion, Madela was correct to cite Great Eastern for having performed research without an IACUC protocol. The IACUC Chairperson needs to address the development of an institutional policy to deal with blood donors and similar animal-related activities. Many institutions have developed policies that allow for a "shortened" protocol form that goes through an expedited review. One may argue that requiring an IACUC-approved protocol to use donated blood is yet another way that the burden of paperwork hinders the advancement of science. Because of the diversity of the IACUC members, it is important for a protocol to be reviewed so that the Committee will be able to address the ethical concerns in using humane society animals for research purposes. Did Burbank fail to submit a proposal to the IACUC because he was 'out of time'? This certainly is not a justifiable reason, at least not from the perspective of the law.

\section{References \\ 1. USDA APHIS. 9 CFR, Sec. 2.31. \\ 2. Public Health Service. Policy on Humane Care and Use of Laboratory Animals II (US Department of Health and Human Services, Washington, DC, 1986). \\ 3. Potkay, S., Garnett, N.L., Miller, J.G., Pond, C.L. \& Doyle, D.J. Frequently asked ques- tions about Public Health Service Policy on Humane Care and Use of Laboratory Animals. Lab Anim. (NY) 24, 24-26 (1995). \\ 4. Silverman, J., Suckow, M.A. \& Murthy, S. The IACUC Handbook 8:15 (CRC Press, Boca Raton, FL, 2000). \\ 5. Silverman, J., Suckow, M.A. \& Murthy, S. The IACUC Handbook 14:32 (CRC Press, Boca Raton, FL, 2000).}

Samalonis is Training Coordinator and Lotocki is Assistant Training Coordinator, University Laboratory Animal Resources, University of Pennsylvania, Philadelphia, PA.

\section{Better Safe Than Sorry}

\section{Richard M. Harrison, PhD}

The IACUC Chairperson was correct, in that the drawing of blood for clinical cases does not require an IACUC-approved protocol. If the veterinarian had drawn the blood before Burbank asked for a 1-ml sample, and there was extra blood already drawn, that would have been acceptable. Madela is also correct. One could consider the request for extra blood to be drawn as two separate collections: the usual amount for the clinical studies and the additional $1 \mathrm{ml}$ requested for the research project. Because the additional $1 \mathrm{ml}$ was drawn specifically to obtain data for a research proposal, an IACUC-approved protocol was necessary.

A situation similar to the drawing of blood involves the use of archived tissues that are collected at necropsy from animals on an approved project. Years later, another investigator may examine these archived 
materials to provide preliminary data for a proposed project. Although the tissues were not collected for the preliminary data study, they are available for that study. If the investigator had requested access to these tissues before they were collected, then that would require an approved IACUC amendment to the project involving the animals at the time of necropsy. If tissues and blood are routinely collected at necropsy and are later available for preliminary data, then an IACUC-approved protocol should not be necessary.

It is always better to have an IACUC protocol approved before you request tissues or fluids from a living animal that is covered by the Animal Welfare Act (AWA). It is better to have a protocol approved that you don't need than to need one that you don't have.

In the Great Eastern situation, the veterinarian of the humane society could have voluntarily released the dogs, temporarily, to Burbank for the blood draws. Voluntary release is permissible under the AWA ${ }^{1}$. This, however, would have still have required an approved IACUC protocol.

Reference

1. Animal Welfare Act, 7 USC 2157, Sec. 28 (a) (2)(B).

Harrison is Research Scientist, Division of Veterinary Medicine, Tulane National Primate Research Center, Covington, LA. 